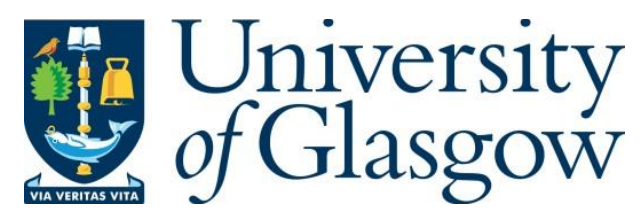

Mansell, R., Petit, D.C.M.C., Fernández-Pacheco, A., Lavrijsen, R., Lee, J.H. and Cowburn, R.P. (2018) Sputter grown $\mathrm{Fe}$ and $\mathrm{Fe} / \mathrm{Cr}$ multilayers with fourfold magnetic anisotropy on GaAs. IEEE Transactions on Magnetics, 54(1), 2000105.

There may be differences between this version and the published version. You are advised to consult the publisher's version if you wish to cite from it.

http://eprints.gla.ac.uk/204486/

Deposited on: 2 December 2019

Enlighten - Research publications by members of the University of Glasgow http://eprints.gla.ac.uk 


\title{
Sputter Grown Fe and Fe/Cr Multilayers With Fourfold Magnetic Anisotropy on GaAs
}

\author{
R. Mansell ${ }^{\circledR}$, D. C. M. C. Petit, A. Fernández-Pacheco, R. Lavrijsen, J. H. Lee, and R. P. Cowburn \\ Cavendish Laboratory, University of Cambridge, Cambridge CB3 OHE, U.K.
}

\begin{abstract}
Thin films of Fe have been epitaxially sputtered on GaAs substrates with native oxide removal prior to the deposition carried out by an Ar ion milling. Films grown at substrate temperatures above $100{ }^{\circ} \mathrm{C}$ show well-defined fourfold anisotropies. The onset of epitaxial growth is accompanied by an increase in the surface roughness with growth occurring in a distinct island-like pattern. The Fe layers show significantly reduced moments, which decrease with increasing temperature. Antiferromagnetic coupling between Fe layers with $\mathrm{Cr}$ spacers was measured in a multilayer with a $\mathrm{Cr}$ thickness of $2.7 \mathrm{~nm}$, around the second antiferromagnetic peak. The magnetic properties of the films are discussed in the context of multilayer storage applications.
\end{abstract}

Index Terms-Magnetic anisotropy, magnetic hysteresis, magnetic multilayers, spintronics.

\section{INTRODUCTION}

$\mathbf{T}$ HE growth of $\mathrm{Fe}$ on GaAs has been widely studied as a model system of ultrathin film magnetism, including the onset of ferromagnetism [1], [2], changes between uniaxial and cubic anisotropy with thickness [2], [3], domain wall motion [4], [5], and Ruderman-Kittel-KasuyaYosida (RKKY) coupling [6], [7]. It is also a system used for studying spin injection into semiconductors, where the relatively large spin polarization of Fe films has been combined with GaAs-based devices for both spin light emitting diode [8], [9] and lateral spin injection and detection experiments [10]. Another technologically relevant area using the thin magnetic films is data storage. For such applications, not only single layers but also multilayer structures with controllable interlayer coupling between the layers are of interest. Recently, it has been proposed to use RKKY coupled stacks to create 3-D data storage devices, where the information is propagated out-of-plane [11]-[14]. The growth of layers with well-defined uniaxial [13], [14] or fourfold anisotropies [15] and RKKY coupling is required for such multilayer devices.

Epitaxial $\mathrm{Fe}$ has been widely grown by molecular beam epitaxy (MBE) on GaAs surfaces prepared by high temperature oxide removal and annealing [1], [3], [8]. Growth by sputtering is a more commercially relevant technique but it is not certain that the same well-defined magnetic properties can be achieved. Previously, the epitaxial growth of $\mathrm{Fe}$ by sputtering has been obtained on oxide substrates, such as sapphire, using metal underlayers grown at high temperature before Fe growth at lower temperature [16]-[18]. In this way, controlled multilayer stacks demonstrating surface spin-flop and soliton states have been made. Whilst this method allows the growth of controlled multilayers, spintronic applications may also require a high-quality interface with GaAs [8]. Fe has been sputtered epitaxially on GaAs using either high temperatures [19], [20] or very low sputtering rates [21].

Manuscript received May 23, 2016; revised November 8, 2016 and February 28, 2017; accepted September 11, 2017. Corresponding author: R. Mansell (e-mail: rhodri.mansell@aalto.fi).

Digital Object Identifier 10.1109/TMAG.2017.2755584
Fe grown on GaAs is known to intermix at relatively low temperatures (around $150{ }^{\circ} \mathrm{C}$ ) although post-growth annealing at these temperatures can improve the quality of the interface [22]. Previous growths of $\mathrm{Fe}$ on GaAs using sputtering have been able to show both epitaxy and low ferromagnetic resonance linewidths [23], [24].

In this paper, the growth of single Fe layers as well as $\mathrm{Fe} / \mathrm{Cr}$ multilayers grown epitaxially by sputtering on GaAs is studied. We look at optimizing the growth of single layers in terms of the anisotropies of the layers, particularly, how close they are to an ideal fourfold anisotropy. We also study multilayers in terms of the achievable RKKY coupling. For multilayer data storage, it is particularly important to be able to reproduce the anisotropy and coupling on a layer-by-layer basis.

\section{Single FE LAYERS}

Samples were grown by magnetron sputtering in a system with a base pressure of $3 \times 10^{-8}$ mbar using an Ar gas pressure of $8 \times 10^{-3}$ mbar. $\mathrm{Fe}$ and $\mathrm{Cr}$ films were deposited at a power of $50 \mathrm{~W}$, with the deposition rates of around $0.06 \mathrm{~nm} / \mathrm{s}$. No in situ characterization techniques are available. Semi-insulating GaAs(100) wafers were used as substrates. Untreated GaAs wafers have a native oxide present at the surface. Fe layers grown directly onto the GaAs with the native oxide still present show a polycrystalline behavior even at elevated growth temperatures. The oxide can be removed by chemical means, for example, etching in $\mathrm{HCl}$. However, the native oxide will rapidly reform, requiring this procedure to be carried out immediately before the introduction of the wafer into the vacuum system. It is, therefore, preferable to use an in situ technique to remove the oxide. Here, the native oxide was removed by an Ar ion milling [19]. The milling system is located in the same chamber as the sputtering magnetrons, which allows for a very small delay between the milling and the start of the Fe growth, so that little oxide will reform on the surface even at the higher base pressures used here in comparison with MBE systems. This higher base pressure is also why annealing was not carried out prior to growth. A gridded dc ion milling system with an acceleration 
TABLE I

Growth Temperature Dependence of Structural and Magnetic Properties of Fe Single Layers

\begin{tabular}{|c|c|c|c|c|c|c|}
\hline Sample & $\begin{array}{c}\text { Thickness } \\
(\mathrm{nm})\end{array}$ & $\begin{array}{c}\text { Ion } \\
\text { milling } \\
\text { time }(\mathrm{s})\end{array}$ & $\begin{array}{c}\text { Growth } \\
\text { temperature } \\
\left({ }^{\circ} \mathrm{C}\right)\end{array}$ & $\begin{array}{c}\text { Growth } \\
\text { pressure } \\
\text { mbar }\end{array}$ & $\begin{array}{c}\text { Ms } \\
(\mathrm{emu} / \mathrm{cc})\end{array}$ & $\begin{array}{c}\text { rms } \\
\text { roughness } \\
(\mathrm{nm})\end{array}$ \\
\hline $\mathrm{A}$ & 12 & 15 & 20 & $1.5 \times 10^{-7}$ & 790 & 0.3 \\
\hline $\mathrm{B}$ & 12 & 15 & 50 & $2.3 \times 10^{-7}$ & 710 & 0.7 \\
\hline $\mathrm{C}$ & 12 & 15 & 100 & $2.6 \times 10^{-7}$ & 690 & 1.2 \\
\hline $\mathrm{D}$ & 12 & 15 & 150 & $3.4 \times 10^{-7}$ & 520 & 1.0 \\
\hline
\end{tabular}
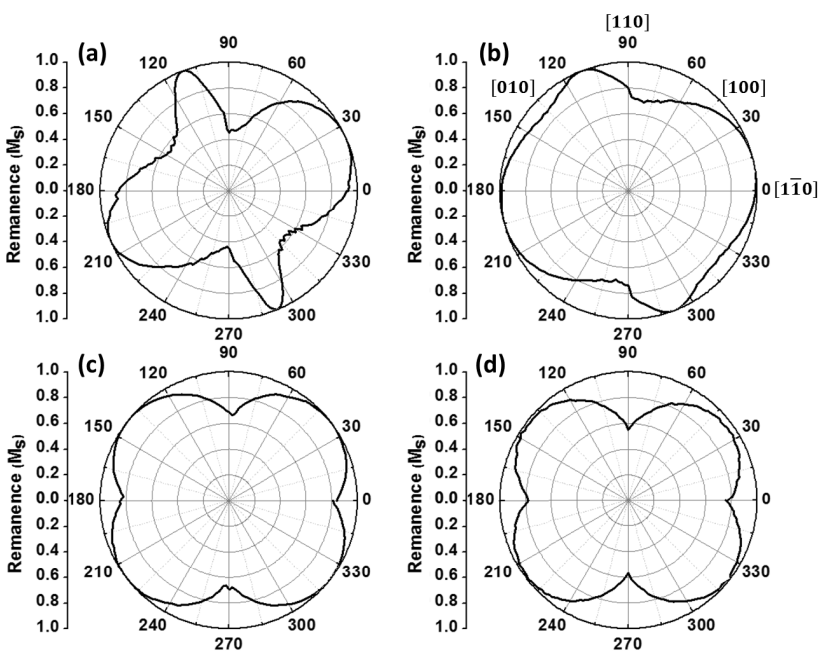

Fig. 1. Normalized angular remanent moment plots as a function of in-plane angle for $12 \mathrm{~nm}$ thick Fe samples grown at (a) $20^{\circ} \mathrm{C}$, (b) $50{ }^{\circ} \mathrm{C}$, (c) $100{ }^{\circ} \mathrm{C}$, and (d) $150{ }^{\circ} \mathrm{C}$. (b) also shows the crystallographic directions of the GaAs substrate.

voltage of $350 \mathrm{~V}$ and $28 \mathrm{~mA}$ beam current was used, with the beam at normal incidence to the sample. The ion milling parameters were kept constant for all growths. The magnetic properties of layers were characterized for different milling times and it was found that $15 \mathrm{~s}$, which corresponds to around $3 \mathrm{~nm}$ of milling, was optimal for Fe growth and is used for all the growths presented here. This would correspond to the thickness range of native oxide found on GaAs surfaces exposed to air for several years, which has been found to be between 2 and $3 \mathrm{~nm}$ [25]. For shorter milling times the layers do not show fourfold anisotropy $(5 \mathrm{~s})$ or have reduced anisotropy (10 s), whilst at longer times (20 s) a reduced anisotropy is seen (not shown here). This behavior is likely due to not fully removing the oxide for shorter milling times and due to increased roughness on the surface caused by the milling for longer times. A series of four $12 \mathrm{~nm}$ thick Fe layers were grown on GaAs substrates at different temperatures, which were ion milled for $15 \mathrm{~s}$ immediately prior to growth. The Fe layers were then capped with Cr. Structural and magnetic properties of the films are listed in Table I. The growth pressure refers to the background pressure of the chamber with the substrate at growth temperature immediately prior to deposition.

\section{A. Magnetic Properties}

Fig. 1 shows the angular remanent moment measurements of the four films. The maxima give the positions of the easy axes whilst minima define the hard axes. It is apparent that there is a change in the anisotropy of the films with increasing growth temperature. For all the measurements here, the [11 10$]$ direction of the GaAs is aligned along $0^{\circ}$. For the $20^{\circ} \mathrm{C}$ and $50{ }^{\circ} \mathrm{C}$ films, the anisotropy is a mix of uniaxial and fourfold terms. The uniaxial term easy axis appears to be at around $30^{\circ}$, between the [110] and [100] axes of the GaAs. For MBE grown Fe films on (001) GaAs two regimes of anisotropy with thickness are observed [3]. For films a few monolayers thick the anisotropy is dominated by an interfacial uniaxial term, which has an easy axis along the [110] direction [3]. For thicker films, the effect of this interface term is reduced and the films are dominated by the fourfold anisotropy term expected from the crystallography. The films studied here are $12 \mathrm{~nm}$ thick, which is beyond the thickness, where a uniaxial term is expected to dominate, assuming its origin at the GaAs interface.

At $100{ }^{\circ} \mathrm{C}$ and $150{ }^{\circ} \mathrm{C}$, the samples acquire a dominant fourfold anisotropy. However, an asymmetry still remains between the two hard axes along the [1 10$]\left(0^{\circ}\right)$ and $[110]\left(90^{\circ}\right)$ directions. Vibrating sample magnetometer (VSM) hysteresis loops are shown in Fig. 2, for the [110], [110], and [100] directions of sample D (see Table I). The saturation along the [1110] direction occurs at around 300 Oe, whilst that for the [110] direction occurs at around 500 Oe. For bulk Fe, the fourfold anisotropy gives a saturation field of 550 Oe. These results suggest the presence of a bulk uniaxial term in the Fe layers, with an easy axis along the [110] direction $\left(0^{\circ}\right)$. Such a term has been found before and shown to be along the [110] direction [26]. It is possible that this is also seen in the $20{ }^{\circ} \mathrm{C}$ and $50{ }^{\circ} \mathrm{C}$ data, which could be explained by combining a uniaxial term along [1 10$]$ and a fourfold easy axis along the [100] direction, so causing the apparent intermediate easy direction found experimentally. The existence of the uniaxial term has been ascribed to defects in the bulk of the film, which may be more likely in sputter growth than in typical MBE films [27]. However, the low ( $<10$ Oe) coercivity along the easy axes of the films [see Fig. 2(c)] suggests that the defects are not impeding domain wall motion through the films, as has been suggested as a sign of a high density of impurities in the bulk [27].

The saturation magnetization of the films was also extracted from VSM measurements, and is listed in Table I. It is notable how reduced these values are from the expected $1760 \mathrm{emu} / \mathrm{cm}^{3}$ found in bulk as well as MBE grown films [2]. The saturation magnetization also decreases with the growth temperature. From MBE studies, it is known that growth at 

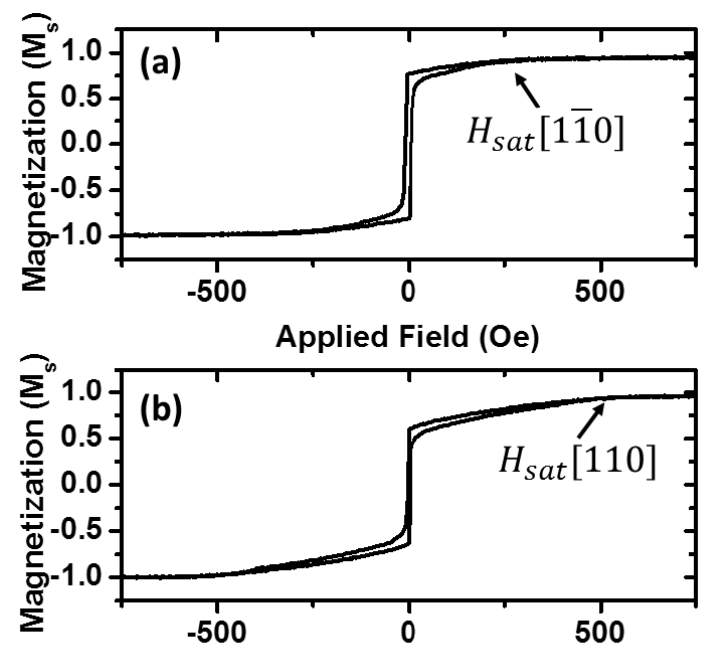

Applied Field (Oe)

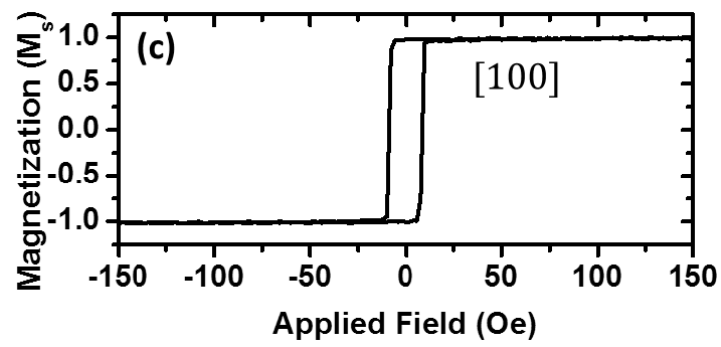

Fig. 2. VSM hysteresis loops taken on sample D at (a) $0^{\circ}$ ([110]), (b) $90^{\circ}$ ([110]), and (c) $45^{\circ}$ ([100]).

raised temperatures may lead to reduced magnetization due to the intermixing of the Fe with the GaAs [28]. This may explain why the saturation magnetization decreases at higher temperatures, but this is on top of the significantly reduced $M_{s}$ even at room temperature. This may be due to roughness, defects, and inclusions in the film as well as possibly oxidation due to the high background pressure of the chamber combined with the relatively slow rate of growth. Using the $M_{s}$ values obtained, the effective anisotropy constants can be extracted using $K_{1}=M_{S} H_{\text {sat }} / 2$. Using the data in Table I and Fig. 2, the fourfold anisotropy terms along the [1ํㅣ and [110] directions are $0.8 \times 10^{5} \mathrm{erg} / \mathrm{cm}^{3}$ and $1.3 \times 10^{5} \mathrm{erg} / \mathrm{cm}^{3}$, respectively, compared with an expected $4.8 \times 10^{5} \mathrm{erg} / \mathrm{cm}^{3}$ for bulk anisotropy [2]. Most simply the difference between the two hard axis anisotropies could be explained by the presence of a uniaxial term with an easy axis along the [110] direction. This would give a fourfold-term of $0.8 \times 10^{5} \mathrm{erg} / \mathrm{cm}^{3}$ and a uniaxial term of $0.5 \times 10^{5} \mathrm{erg} / \mathrm{cm}^{3}$ in this sample.

\section{B. Surface Properties}

Contact atomic force microscopy (AFM) on the single layer Fe samples was carried out, with images obtained for samples $\mathrm{A}$ and $\mathrm{C}$ as shown in Fig. 3(a) and (c), respectively. Single line scans for these samples are shown for sample A in Fig. 3(b) and for sample $\mathrm{C}$ in Fig. 3(d). For the samples grown at room temperature [Fig. 3(a)], a smooth surface is found with rms roughness around $0.3 \mathrm{~nm}$, which is the same as that found for a GaAs substrate after $15 \mathrm{~s}$ of ion milling. As the temperature is
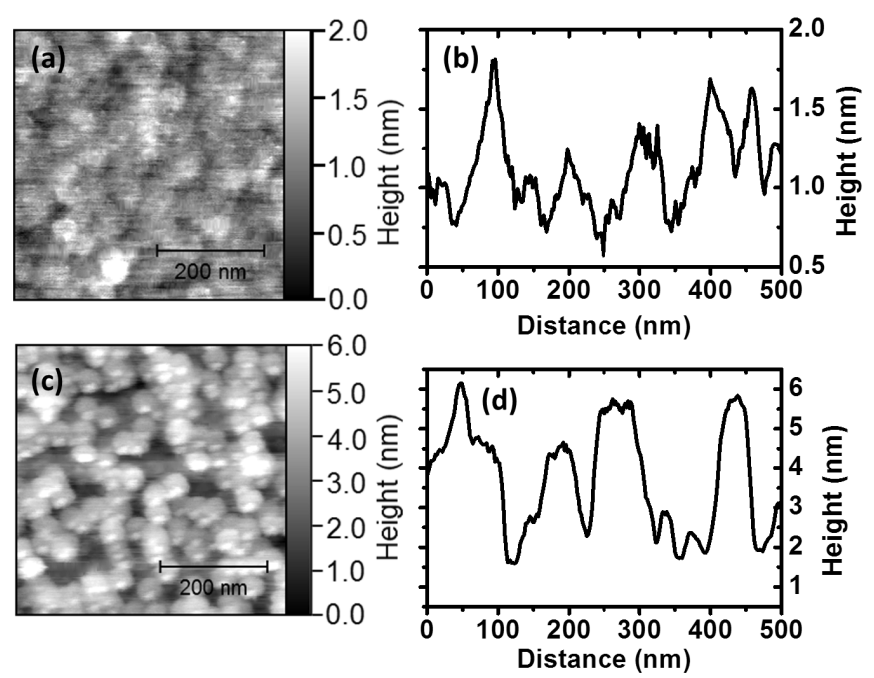

Fig. 3. Contact AFM images of samples (a) A and (c) C, along with line scans for (b) A and (d) C. Note the differences in vertical scale.

increased, the roughness increases and the epitaxial samples have a distinctly textured surface with increased roughness as shown for sample $\mathrm{C}$ in Fig. 3(c). The surface appears to consist of circular columns several nanometers high, with no apparent tendency for growth along any of the underlying GaAs crystallographic directions. The same circular features are visible in the non-epitaxial sample, although much less prominently, which suggests that the texture is not related to the magnetic anisotropy of the films.

\section{Multilayer SAMPLES}

The Fe/Cr system has large RKKY coupling with oscillations over two different periods caused by the antiferromagnetism of the $\mathrm{Cr}$ [7]. For sputtered $\mathrm{Fe} / \mathrm{Cr}$ multilayers the long-wavelength oscillation has been reported as a function of temperature [29].

Multilayer $\mathrm{Fe} / \mathrm{Cr}$ superlattices were grown on an Fe $(12 \mathrm{~nm}) / \mathrm{Cr}(15 \mathrm{~nm})$ buffer layer. The initial Fe buffer layer ensures the epitaxy of the growth, however, this layer may intermix with the GaAs, so a further $\mathrm{Cr}$ buffer is used before the multilayer growth. Shown in Fig. 4 are the hard [Fig. 4(a)] and easy [Fig. 4(b)] axis loops of a $[\mathrm{Fe}(1.5 \mathrm{~nm}) / \mathrm{Cr}(2.7 \mathrm{~nm})]_{9} / \mathrm{Fe}(1.5 \mathrm{~nm}) / \mathrm{Cr}(4.5 \mathrm{~nm})$ multilayer. The loops show a slow saturation of the magnetization up to around $6000 \mathrm{Oe}$, in contrast to the behavior shown in Fig. 2. This is due to the extra field required to overcome the antiferromagnetic coupling between layers. The thickness of $\mathrm{Cr}$ used is around that expected for the second antiferromagnetic peak in $\mathrm{Cr}(001)$ layers [29]. The data also include the moment from the $12 \mathrm{~nm} \mathrm{Fe}$ underlayer, which is shown in the low field data in the insets of Fig. 4. Here, the distinction between the easy and hard axis data, which mainly reflects the magnetization of the $12 \mathrm{~nm}$ Fe buffer, is more apparent, as seen in the higher remanence and more coercive switching in the easy axis direction.

Shown in Fig. 4(c) is the remanent magnetization after saturation as a function of angle, which shows a remanant 

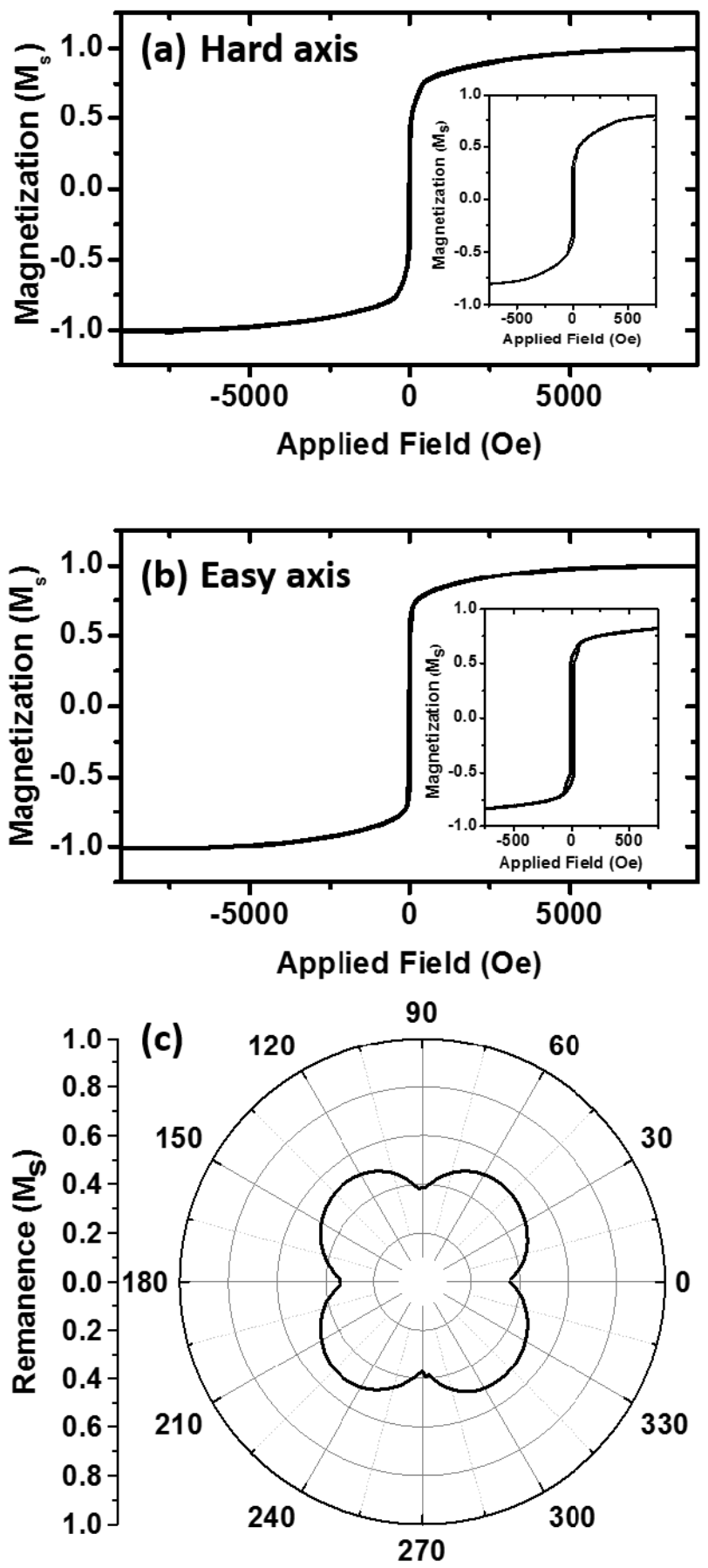

Fig. 4. Normalized VSM loops of an Fe/Cr multilayer sample. (a) [11̄0] GaAs direction. (b) [100] GaAs direction. Insets: low field behavior. (c) Remanant magnetization of the sample.

moment of about half the saturation moment with a clear fourfold anisotropy. This is probably due entirely to the Fe underlayer. Assuming the Fe underlayer has the same saturation magnetization as the single layer measured previously, the saturation magnetization of the $10 \times 1.5 \mathrm{~nm}$ Fe multilayer can be calculated as $700 \mathrm{emu} / \mathrm{cm}^{3}$, which is similar to that found in the single layer samples. From this an antiferromagnetic coupling strength of around $-0.15 \mathrm{erg} / \mathrm{cm}^{2}$ can be obtained. This is slightly larger than has been obtained previously [30], but is dependent on the assumptions made concerning the saturation magnetization of the layers. A large coupling would also be consistent with the lack of anisotropy, as shown in Fig. 4(c). However, multilayers with thicknesses around the first antiferromagnetic peak at $1 \mathrm{~nm}$ in $\mathrm{Cr}$ did not show any RKKY coupling, and neither did those around the third antiferromagnetic peak at $4.5 \mathrm{~nm}$, both giving results consistent with strongly ferromagnetically coupled layers. This may be due to the roughness of the samples, leading to discontinuous layers for the thin $\mathrm{Cr}$ thicknesses. The sample that showed coupling had a large rms roughness of $4 \mathrm{~nm}$ and the other multilayer growths showed roughnesses between 2 and $4 \mathrm{~nm}$.

\section{DISCUSSION}

The possibility of using sputtered $\mathrm{Fe}$ on GaAs for spintronic applications has previously been demonstrated, at least at low temperature [21], [31]. Notably, these examples use ultrahigh vacuum sputtering, which allows low sputtering rates to be used without oxidizing the interface. The high temperatures, and consequently high background pressures, required to create epitaxial samples here may mitigate against the use of this devices in spintronic applications due to the likelihood of increased interfacial intermixing and oxidation. The surface preparation process here also may not favor spintronic applications compared with in situ transfer techniques, which can produce sharp clean interfaces [8], [9], [32]. The strongly reduced $M_{S}$ also suggests that the spin polarization of such devices would be significantly reduced.

For use in magnetic multilayers, a reduction in $M_{s}$ is not problematic as long as it is fairly consistent across the multilayer. It was found that this was the case for the multilayers studied, where the magnetization of the ten times repeats was consistent with that of a single layer. More problematically, it appears that the roughness and column-like growth may lead to a limited range of available coupling strength. The sample shown in Fig. 4 is the only one, grown in the same conditions whilst varying the $\mathrm{Cr}$ thickness that showed RKKY coupling. Previously, oscillatory RKKY coupling in sputtered multilayers has been found in epitaxial [33] and nonepitaxial layers [29]. The roughness in the multilayers studied here may be due to the high temperatures used to establish the epitaxial growth of $\mathrm{Fe}$ on GaAs. For proposed multilevel memory applications, where each layer has reproducible properties [11]-[15] the use of high temperature buffer layers to provide a smooth metallic surface for the $\mathrm{Fe} / \mathrm{Cr}$ epitaxy may be preferential, which can be achieved by using, for instance, $\mathrm{MgO}$ substrates [18], [33].

\section{CONCLUSION}

A series of single $\mathrm{Fe}$ and multilayer $\mathrm{Fe} / \mathrm{Cr}$ films have been grown by magnetron sputtering on GaAs substrates prepared using an argon ion milling. Epitaxial growth of the $\mathrm{Fe}$ is achieved for temperatures above $100{ }^{\circ} \mathrm{C}$, where a clear fourfold magnetization is found. Epitaxial layers show significant roughness and all layers show a reduction in saturation magnetization. An $\mathrm{Fe} / \mathrm{Cr}$ multilayer sample showed significant antiferromagnetic coupling, where the $\mathrm{Cr}$ 
thickness was around the second antiferromagnetic peak. However, despite the promising magnetic properties of the samples, the roughness of the layers most likely precludes the use of this materials system for in multilevel memory applications.

\section{ACKNOWLEDGMENT}

This work was supported in part by the European Community through the Seventh Framework Program ERC under Contract 247368: 3SPIN and in part by the Netherlands Organization for Scientific Research and Marie Curie Cofund Action under Grant NWO-Rubicon 680-50-1024.

\section{REFERENCES}

[1] Y. B. Xu, E. T. M. Kernohan, D. J. Freeland, A. Ercole, M. Tselepi, and J. A. C. Bland, "Evolution of the ferromagnetic phase of ultrathin Fe films grown on GaAs(100)-4×6," Phys. Rev. B, Condens. Matter, vol. 58, pp. 890-896, Jul. 1998.

[2] G. Wastlbauer and J. A. C. Bland, "Structural and magnetic properties of ultrathin epitaxial fe films on $\operatorname{GaAs}(001)$ and related semiconductor substrates," Adv. Phys., vol. 54, no. 2, pp. 137-219, 2005.

[3] M. Gester, C. Daboo, R. J. Hicken, S. J. Gray, A. Ercole, and J. A. C. Bland, "Continuous evolution of the inplane magnetic anisotropies with thickness in epitaxial Fe films," J. Appl. Phys., vol. 80, no. 1, pp. 347-355, 1996.

[4] R. P. Cowburn, S. J. Gray, and J. A. C. Bland, "Multijump magnetic switching in in-plane magnetized ultrathin epitaxial Ag/Fe/Ag(001) films," Phys. Rev. Lett., vol. 79, pp. 4018-4021, Nov. 1997.

[5] N.-J. Steinke, T. A. Moore, R. Mansell, J. A. C. Bland, and C. H. W. Barnes, "Nonuniversal dynamic magnetization reversal in the Barkhausen-dominated and mesofrequency regimes," Phys. Rev. B, Condens. Matter, vol. 86, pp. 184434-1-184434-8, Nov. 2012.

[6] A. J. R. Ives, J. A. C. Bland, R. J. Hicken, and C. Daboo, "Oscillatory biquadratic coupling in fe/cr/fe(001)," Phys. Rev. B, Condens. Matter, vol. 55, p. 12428, May 1997.

[7] J. Unguris, R. J. Celotta, and D. T. Pierce, "Observation of two different oscillation periods in the exchange coupling of $\mathrm{Fe} / \mathrm{Cr} / \mathrm{Fe}(100)$," Phys. Rev. Lett., vol. 67, pp. 140-143, Jul. 1991.

[8] A. T. Hanbicki, B. T. Jonker, G. Itskos, G. Kioseoglou, and A. Petrou, "Efficient electrical spin injection from a magnetic metal/tunnel barrier contact into a semiconductor," Appl. Phys. Lett., vol. 80, no. 7, pp. 1240-1242, 2002

[9] R. Mansell et al., "Ingaas spin light emitting diodes measured in the Faraday and oblique Hanle geometries," J. Phys. D, Appl. Phys., vol. 49, no. 16, p. 165103, 2016.

[10] X. Lou et al., "Electrical detection of spin transport in lateral ferromagnet-semiconductor devices," Nature Phys., vol. 3, pp. 197-202, Feb. 2007.

[11] R. Lavrijsen, J.-H. Lee, D. C. M. C. Petit, A. Fernández-Pacheco, R. Mansell, and R. P. Cowburn, "Magnetic ratchet for three-dimensional spintronic memory and logic," Nature, vol. 493, pp. 647-650, Jan. 2013.

[12] R. Lavrijsen, D. C. Petit, A. Fernández-Pacheco, J. Lee, R. Mansell, and R. P. Cowburn, "Multi-bit operations in vertical spintronic shift registers," Nanotechnology, vol. 25, p. 105201, Feb. 2014.

[13] D. Petit, R. Mansell, A. Fernández-Pacheco, J. Lee, and R. P. Cowburn, Three-Dimensional Spintronics (Devices, Circuits, and Systems). Boca Raton, FL, USA: CRC Press, 2014, ch. 12.
[14] A. Fernández-Pacheco, D. Petit, R. Mansell, R. Lavrijsen, J. H. Lee, and R. P. Cowburn, "Controllable nucleation and propagation of topological magnetic solitons in CoFeB/Ru ferrimagnetic superlattices," Phys. Rev. B, Condens. Matter, vol. 86, p. 104422, Sep. 2012.

[15] R. Mansell et al., "Magnetic properties and interlayer coupling of epitaxial Co/Cu films on Si," J. Appl. Phys., vol. 116, no. 6, p. 063906, 2014.

[16] R. W. Wang, D. L. Mills, E. E. Fullerton, J. E. Mattson, and S. D. Bader, "Surface spin-flop transition in $\mathrm{Fe} / \mathrm{Cr}(211)$ superlattices: Experiment and theory," Phys. Rev. Lett., vol. 72, pp. 920-923, Feb. 1994.

[17] M. Grimsditch, S. Kumar, and E. E. Fullerton, "Brillouin light scattering study of $\mathrm{Fe} / \mathrm{Cr} / \mathrm{Fe}$ (211) and (100) trilayers," Phys. Rev. B, Condens. Matter, vol. 54, pp. 3385-3393, Aug. 1996.

[18] J. Meersschaut et al., "Hard-axis magnetization behavior and the surface spin-flop transition in antiferromagnetic Fe/Cr(100) superlattices," Phys. Rev. B, Condens. Matter, vol. 73, p. 144428, Apr. 2006.

[19] F. Monteverde, A. Michel, P. Guérin, and J.-P. Eymery, "Epitaxial growth of fe on GaAs by ion beam sputtering," Surf. Sci., vol. 482, pp. 872-877, Jun. 2001.

[20] F. Monteverde, A. Michel, J.-P. Eymery, and P. Guérin, "Structure characterization of fe films grown on GaAs $\left(\begin{array}{lll}1 & 0 & 0\end{array}\right)$ by ion-beam sputter epitaxy," J. Cryst. Growth, vol. 267, nos. 1-2, pp. 231-238, 2004.

[21] E. Wada, M. Itoh, and T. Taniyama, "Crossover of electron transmission mechanism and spin filtering effect at Fe/GaAs(001) interfaces," J. Appl. Phys., vol. 103, no. 7, p. 07A702, 2008.

[22] S. Park, M. R. Fitzsimmons, C. F. Majkrzak, B. D. Schultz, and C. J. Palmstrøm, "The influence of growth temperature and annealing on the magnetization depth profiles across ferromagnetic/semiconductor interfaces," J. Appl. Phys., vol. 104, no. 8, p. 083905, 2008.

[23] S. Kreuzer, K. Prügl, G. Bayreuther, and D. Weiss, "Epitaxial growth and patterning of sputtered Fe films on GaAs(001)," Thin Solid Films, vol. 318, nos. 1-2, pp. 219-222, 1998.

[24] Y. Takahashi, H. Ikeya, N. Inaba, F. Kirino, M. Ohtake, and M. Futamoto, "Q-band ferromagnetic resonance study of Fe thin films on GaAs(001) deposited by RF magnetron sputtering," IEEE Trans. Magn., vol. 47, no. 12, pp. 4682-4685, Dec. 2011.

[25] C. W. Wilmsen, Oxide/III-V Compound Semiconductor Interfaces. New York, NY, USA: Plenum, 1985, ch. 7.

[26] G. Chen, J. Zhu, J. Li, F. Z. Liu, and Y. Z. Wu, "Revealing the volume magnetic anisotropy of Fe films epitaxied on GaAs(001) surface," Appl. Phys. Lett., vol. 98, no. 13, p. 132505, 2011.

[27] G. Bayreuther, J. Premper, M. Sperl, and D. Sander, "Uniaxial magnetic anisotropy in Fe/GaAs(001): Role of magnetoelastic interactions," Phys. Rev. B, Condens. Matter, vol. 86, p. 054418, Aug. 2012.

[28] J. J. Krebs, B. T. Jonker, and G. A. Prinz, "Properties of Fe single-crystal films grown on (100)GaAs by molecular-beam epitaxy," J. Appl. Phys., vol. 61, no. 7, pp. 2596-2599, 1987.

[29] S. S. Parkin, N. More, and K. P. Roche, "Oscillations in exchange coupling and magnetoresistance in metallic superlattice structures: $\mathrm{Co} / \mathrm{Ru}$, Co/Cr, and Fe/Cr," Phys. Rev. Lett., vol. 64, pp. 2304-2307, May 1990.

[30] E. E. Fullerton, M. J. Conover, J. E. Mattson, C. H. Sowers, and S. D. Bader, "Oscillatory interlayer coupling and giant magnetoresistance in epitaxial Fe/Cr(211) and (100) superlattices," Phys. Rev. B, Condens. Matter, vol. 48, p. 15755, Dec. 1993.

[31] J. Moser, A. Matos-Abiague, D. Schuh, W. Wegscheider, J. Fabian, and D. Weiss, "Tunneling anisotropic magnetoresistance and spin-orbit coupling in Fe/GaAs/Au tunnel junctions," Phys. Rev. Lett., vol. 99, p. 056601, Aug. 2007.

[32] J. Moser et al., "Bias dependent inversion of tunneling magnetoresistance in Fe/GaAs/Fe tunnel junctions," Appl. Phys. Lett., vol. 89, no. 16, p. 162106, 2006.

[33] E. E. Fullerton, M. J. Conover, J. E. Mattson, C. H. Sowers, and S. D. Bader, " $150 \%$ magnetoresistance in sputtered Fe/Cr(100) superlattices,” Appl. Phys. Lett., vol. 63, no. 12, pp. 1699-1701, 1993. 